\title{
Têtes de lecture et mémoires magnétiques
}

Jean-Pierre Nozières (jpnozieres@crocus-technology.com)

Crocus Technology, 5 place Robert Schuman, 38025 Grenoble Cedex

Cet article présente deux applications de l'électronique de spin qui ont ou vont révolutionner notre quotidien.

Les têtes de lecture pour disques durs, qui équipent maintenant tous les ordinateurs. C'est le composant clef qui a permis I'accroissement spectaculaire au cours des dix dernières années de la capacité de stockage informatique, à l'origine de la révolution numérique.

Les mémoires magnétiques

à accès aléatoire (MRAM), qui pourraient permettre d'intégrer sur une même puce les fonctions logiques et une mémoire combinant vitesse, endurance et non-volatilité.

Des efforts importants doivent cependant encore être menés pour résoudre les problèmes techniques auxquels se heurte toute nouvelle technologie avant de pouvoir être industrialisée en volume.
L'utilisation de phénomènes physiques nouveaux dans des dispositifs commerciaux est souvent longue à mettre en œuvre, en raison de l'inadéquation entre les performances «théoriques " revendiquées par les chercheurs, estimées dans des conditions idéales de fonctionnement, et les besoins " réels " des entreprises qui impliquent souvent des compromis liés aux conditions de fonctionnement et aux contraintes économiques. L'électronique de spin, c'està-dire la convergence du nanomagnétisme et de l'électronique silicium, a démontré au cours des vingt dernières années qu'avec un peu de chance et beaucoup d'efforts, le transfert pouvait être très rapide.

\section{Les débuts:}

\section{les têtes de lecture pour disques durs}

\section{Historique}

Depuis l'émergence des premiers disques durs au milieu des années 50, la technologie de lecture/écriture des données était restée immuable, basée sur le principe de l'électro-aimant : un courant dans une bobine génère un champ magnétique (pour écrire), le champ magnétique rayonné par les domaines magnétiques écrits dans le support magnétique génère, dans une bobine, un courant induit (pour lire). Seule la technologie de fabrication de ces têtes de lecture dites "inductives » a évolué au cours du temps, passant d'électro-aimants patiemment bobinés à la main sur des noyaux macroscopiques de ferrite, aux têtes intégrées réalisées de manière massivement parallèle en technologie microélectronique.

Avec l'explosion du numérique et les besoins croissants en capacité de stockage, les têtes inductives ont rapidement atteint leurs limites : en effet, lorsque les dimensions des « bits " individuels deviennent trop petites, la quantité de flux magnétique issu du support d'enregistrement est trop faible pour pouvoir être captée efficacement par la bobine.

La découverte de la magnétorésistance géante en 1988, rapidement suivie de la mise au point des "vannes de spin" (voir Reflets $\mathrm{n}^{\circ} 16$, p. 6), version utilisable à

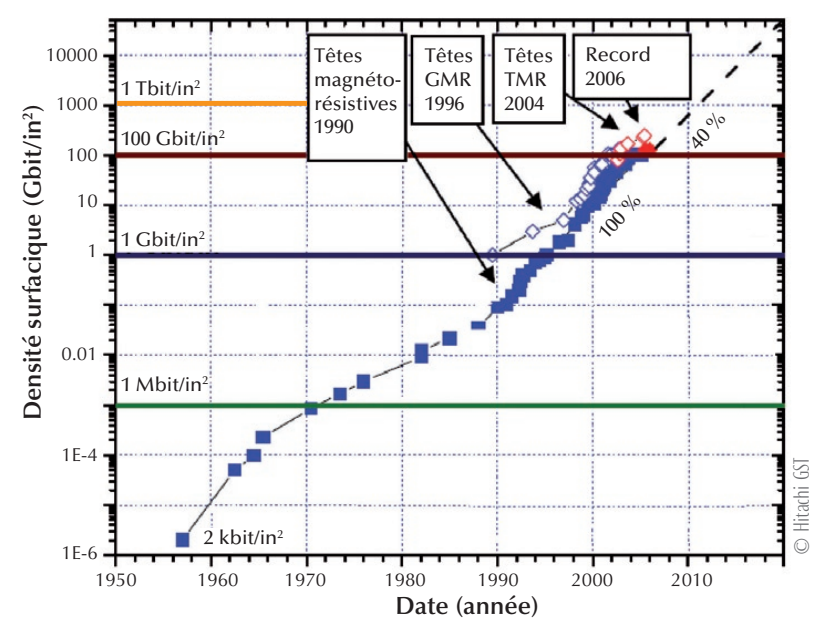

1. Évolution de la densité de stockage des disques durs avec le temps, mettant en évidence les ruptures technologiques. $\diamond, \diamond$ : démonstrations de laboratoire - $\mathbf{\square}, \mathbf{\square}$ : produits commerciaux.

En bleu : technologie traditionnelle « longitudinale », où l'aimantation des domaines magnétiques d'enregistrement des données est parallèle au plan du disque (fig. 2b). En rouge : technologie PMR, commercialisée en 2006, où l'aimantation des domaines est perpendiculaire au plan du disque, ce qui permet d’augmenter la densité de stockage. 


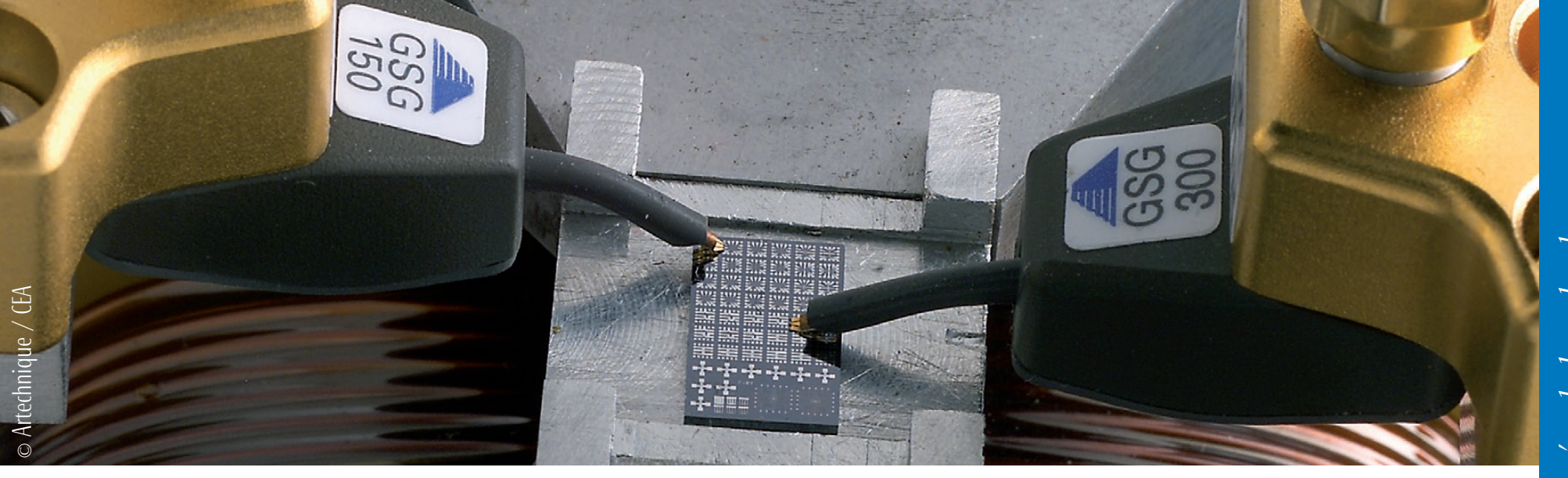

Banc de mesure, au centre CEA de Grenoble, pour la caractérisation de la variation de résistance électrique de composants spintroniques.

température ambiante et présentant une réponse linéaire en champ faible, a complètement redistribué les cartes. La GMR promettait en effet simultanément un gain immédiat en performances (sensibilité à la lecture et fréquence d'opération très supérieures aux meilleures têtes inductives), une miniaturisation possible jusqu'aux dimensions nanométriques (l'effet de magnétorésistance géante étant a priori indépendant de la taille du capteur) et une intégration facilitée par l'accord d'impédance avec les préamplificateurs des circuits de lecture existants.

Moins de dix ans après la découverte de l'effet physique dans un laboratoire de recherche fondamentale, les premiers produits commerciaux étaient vendus au grand public et, en quelques années seulement, $100 \%$ des disques durs étaient équipés de têtes de lecture GMR, soit un marché annuel de $\sim 5$ milliards de $\$$. Aujourd'hui, la technologie GMR est progressivement remplacée par la technologie TMR à base de jonctions tunnel (voir Reflets $\mathrm{n}^{\circ} 16$, p. 8), qui présente une magnétorésistance plus élevée et donc in fine une sensibilité supérieure (fig. 1).

Ce virage technologique a eu une conséquence économique indirecte. Bien que la technologie de fabrication et le mode opératoire au niveau système soient sensiblement identiques, la physique sousjacente et donc les problèmes à résoudre sont fondamentalement différents de ceux rencontrés dans les têtes inductives. En conséquence, seules les premières entreprises ayant réussi le virage technologique de la GMR ont réussi à capturer le marché ; d'où une consolidation spectaculaire du secteur avec, à ce jour, seulement quatre fabricants de têtes de lecture de par le monde - aucun en Europe - dont trois sont verticalement intégrés, fabricant le disque dur complet (Seagate, Hitachi, WD) et un seul encore indépendant (TDK/Headway).

\section{Fonctionnement d'une tête de lecture/ écriture magnétorésistive}

Une tête GMR est formée d'une tête d'écriture et d'une tête de lecture indépendantes dans leur fonctionnement, mais accolées l'une à l'autre et fabriquées simultanément sur le même substrat qui forme le patin de vol. L'ensemble est monté sur un bras connecté au système de positionnement (fig. 2a). En fonctionnement, le disque tourne à environ 10000 tours $/ \mathrm{min}$, ce qui fait voler la tête par effet aérodynamique à $~ 10 \mathrm{~nm}$ de la surface du disque (dont la rugosité est de l'ordre du $\mathrm{nm}$ ) pendant que les bits de données, dont les dimensions actuelles sont de l'ordre de $25 \times 100 \mathrm{~nm}^{2}$, sont écrits et lus à des fréquences proches du GHz. L'analogie frappante de cet ensemble est qu'en redimensionnant le tout à des dimensions macroscopiques, le système dimensionvitesse est équivalent à un Boeing 747 volant à 1 mètre du sol !!

La tête d'écriture est toujours une tête inductive, c'est-à-dire un électro-aimant, même si sa géométrie est très différente de son ancêtre bobinée. La tête de lecture, pour sa part, intègre soit un élément GMR, soit un élément TMR, placé perpendiculairement à la surface du disque de façon à capter le flux magnétique émis par les bits de données (fig. 2b). L'élément magnétorésistif est placé entre deux écrans magnétiques (blindages) permettant d'écranter le flux issu des bits adjacents dans la direction longitudinale (le long des pistes de données). La résolution transverse (de piste à piste) est simplement définie par la largeur de l'élément magnétorésistif, d'où la nécessité de séparer les pistes par une bande sans données ("guardband"). L'élément magnétorésistif est un petit cube, dont la structure en domaines doit être stabilisée à ses extrémités pour éviter le bruit de Barkhausen (mouvements brutaux de l'aimantation autour de pièges que sont les défauts de surface). La stabilisation est
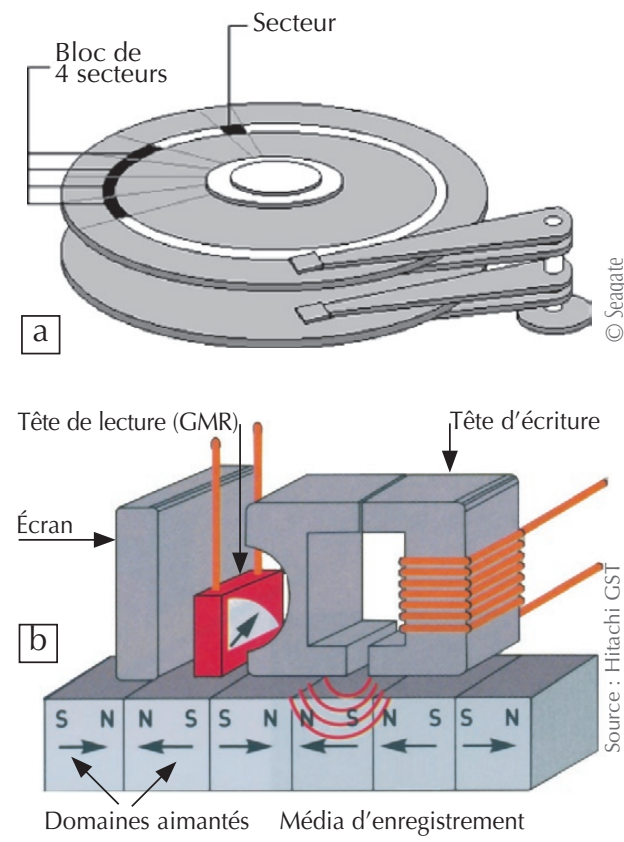

2. Schémas de principe d'un disque dur (a) et d'une tête de lecture/écriture magnétorésistive (b).

effectuée par couplage magnétostatique avec des nano-aimants ("hard bias") adjacents, mais isolés électriquement de l'élément actif.

Dans sa version GMR, le courant de lecture passe dans le plan du capteur (fig. 3a). Des contacts sont pris sur les côtés de l'élément qui doit être isolé électriquement des blindages, d'où des difficultés de fabrication lorsque la densité longitudinale de bits de données est importante, puisque l'espacement entre le blindage et l'élément GMR devient très faible et que des risques de claquage électrique de l'oxyde d'isolation apparaissent. La GMR maximale " théorique " est de l'ordre de $\Delta \mathrm{R} / \mathrm{R}=20 \%$, sachant que la partie « réelle » utilisable, dans laquelle la réponse est linéaire en champ, est d'environ un tiers de cette valeur.

Dans sa version TMR, le courant de lecture circule perpendiculairement au plan du capteur, ce qui permet d'utiliser les blindages comme amenées de courant (fig. 3b). Bien que la TMR maximale 

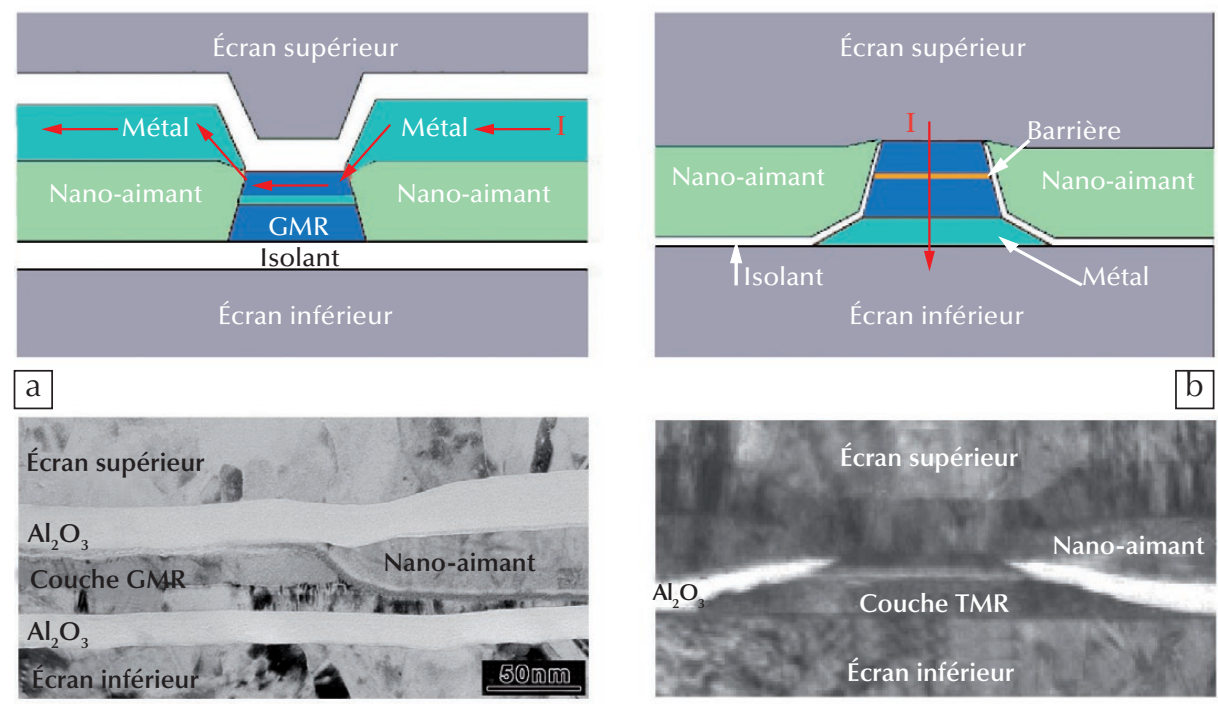

3. Schéma et photo d'une tête GMR (a) et d'une tête TMR (b). Le courant de lecture (I) est indiqué par des flèches rouges.

$\gg$

reportée à ce jour en laboratoire soit de $\sim 1000 \%$, la valeur pratique est limitée à $\Delta \mathrm{R} / \mathrm{R}=100-200 \%$, ce qui est déjà un ordre de grandeur supérieur à la GMR. Ainsi, il est possible aujourd'hui d'atteindre des densités surfaciques de données $>200 \mathrm{Gbit} / \mathrm{in}^{2}$, soit un accroissement de trois ordres de grandeur depuis l'introduction de la GMR il y a 10 ans (fig. 1).

On estime la limite théorique de la technologie actuelle des disques durs aux environs du Tbit/in ${ }^{2}$, à la suite de quoi un changement de paradigme sera nécessaire. Mais le même argument ayant été avancé dans le passé pour des densités de 1, 10 et $100 \mathrm{Gbit} / \mathrm{in}^{2}$, il est possible que la technologie évolue par petites touches, comme c'est le cas, par exemple, pour les mémoires semi-conductrices, dont on annonce régulièrement la mort aux nœuds technologiques «plus » avancés!

\section{Les mémoires magnétiques à accès aléatoire (MRAM)}

\section{Les mémoires RAM actuelles}

Aujourd'hui, la mémoire est partout, avec des capacités allant de quelques kbits dans les appareils électroménagers ou les cartes à puces, aux multi-Gbits dans les baladeurs, les téléphones et les appareils photos. Contrairement aux disques durs qui servent à stocker (archiver) les données et dans lesquels l'accès à celles-ci est séquentiel le long de pistes écrites à la surface du média, les mémoires RAM (Random Access Memories) offrent un accès aléatoire à des bits de données individuels disposés au sein d'un réseau bidimensionnel à l'état solide, c'est-à-dire sans pièces mobiles.

Trois technologies de mémoires RAM semi-conductrices se partagent actuellement le marché, en fonction des principaux paramètres fonctionnels que sont :

- la vitesse d'écriture/lecture ;

- l'endurance, c'est-à-dire sans limitation du nombre de cycles de lecture/écriture possibles ;

- le caractère volatil ou non volatil, c'est-àdire la capacité à conserver l'information en l'absence d'alimentation électrique ;

- la capacité, exprimée en valeur absolue (kbits, Mbits, Gbits, ...), pour l'utilisateur, ou la densité, exprimée en taille de la cellule mémoire élémentaire, pour le concepteur. Cette dernière est souvent normalisée par le carré de la dimension F du nœud technologique de microfabrication utilisé pour sa réalisation (la dimension la plus petite qu'il est possible de fabriquer) ; ceci permet de pouvoir comparer les différentes technologies entre elles indépendamment des contraintes de fabrication. Ainsi, au lieu d'indiquer une dimension de cellule de $90 \times 90 \mathrm{~nm}^{2}$, on dira qu'elle fait $1 \mathrm{~F}^{2}$ en technologie $90 \mathrm{~nm}, 4 \mathrm{~F}^{2}$ en technologie $45 \mathrm{~nm}$, etc ; - la puissance consommée par cycle de lecture/écriture et en mode "standby" ; - le coût de fabrication.

Ainsi, chaque type de mémoire s'est spécialisé, en fonction de ses performances et de ses attributs, à certaines applications spécifiques :

- les SRAM, formées dans leur configuration la plus simple de 4 à 6 transistors CMOS montés en bascule, c'est-à-dire présentant deux états stables, sont très rapides ( $1 \mathrm{~ns})$ et infiniment endurantes $\left(>10^{16}\right.$ cycles), mais volatiles, peu denses $\left(\sim 100 \mathrm{~F}^{2}\right)$ et chères. Ce sont donc les mémoires de choix pour les premiers niveaux de cache (de faible capacité) s'interfaçant directement avec les microprocesseurs ;

- les DRAM, dans lesquelles l'information est stockée sous la forme d'une charge électrique dans une capacité, sont relativement rapides $(\sim 10 \mathrm{~ns})$, denses $\left(\sim 6 \mathrm{~F}^{2}\right)$, endurantes $\left(>10^{16}\right.$ cycles) et de coût raisonnable, mais volatiles. Elles sont donc parfaitement adaptées pour le stockage de données temporaires, dans un ordinateur en aval d'une SRAM et en amont du disque dur. Ce sont aujourd'hui les mémoires les plus répandues, qui représentent $\sim 50 \%$ du marché total en volume ;

- les mémoires Flash, dans lesquelles des charges sont stockées dans une grille flottante localisée au dessus du canal d'un transistor CMOS, sont intrinsèquement non volatiles et de capacité très élevée $\left(<3 \mathrm{~F}^{2}\right)$, mais très lentes $(\sim 100 \mu \mathrm{s})$ et d'endurance limitée $\left(\sim 10^{5}\right.$ cycles en raison du vieillissement de l'oxyde de grille). Elles sont principalement utilisées pour le stockage de code ou de données. Le marché des mémoires est un marché énorme ( 50 milliards \$/ an), dominé par des grosses entreprises comme Samsung et Hynix (Corée), Micron (USA), Toshiba (Japon)..., parmi lesquelles figurent les européens Infineon (Allemagne) et Numonyx, la joint venture récemment créée entre STMicroelectronics et Intel. Mais, contrairement au marché fortement consolidé des disques durs, une multitude d'entreprises coexistent, de la start-up au grand groupe industriel, chacune avec ses spécificités en termes de technologie, d'applications ou de marché (fig. 4).

\section{Les potentialités des mémoires magnétiques}

L'utilisation de cellules magnétiques pour la réalisation de mémoires RAM est une idée ancienne, chaque décennie ayant vu surgir un nouveau concept : les mémoires à base de ferrite dès les années 60 , puis les mémoires à bulles dans les années 80, enfin les mémoires AMR et GMR encore commercialisées par Honeywell, historiquement d'ailleurs les premières MRAM telles qu'on les entend aujourd'hui. Cependant, jusqu'à présent, aucune de ces technologies n'a réussi à percer, en raison de limites physiques de la technologie, de la lenteur du 
développement et surtout de l'essor parallèle spectaculaire des mémoires semi-conductrices.

Le renouveau d'intérêt pour les MRAM provient de la convergence de deux facteurs, l'un technique, l'autre économique :

- intrinsèquement non volatile et infiniment endurante par la nature même du magnétisme, potentiellement rapide $(\sim 10 \mathrm{~ns}$, le temps caractéristique pour faire basculer une aimantation) et miniaturisable jusqu'à des dimensions fortement sub-microniques, la technologie MRAM utilisant des jonctions tunnel magnétiques associe les caractéristiques principales de la SRAM et de la DRAM (vitesse, endurance) avec la non-volatilité de la Flash. À cela se rajoutent des propriétés, telles que l'insensibilité aux rayonnements ionisants, cruciale pour les applications spatiales et militaires, le pilotage par des tensions faibles (contrairement à la Flash) et la miniaturisation théoriquement possible jusqu'aux nœuds technologiques avancés, difficile avec les technologies actuelles;

- l'explosion des besoins, dans des applications de plus en plus spécifiques nécessitant des caractéristiques "à façon ", est actuellement satisfaite par l'association de plusieurs types de circuits logiques et de mémoires, assemblés en composants discrets dans un boîtier unique ou sur une puce unique. Dans ces systèmes complexes et coûteux, une MRAM pourrait ainsi remplacer plusieurs mémoires (généralement SRAM et Flash pour combiner vitesse et non-volatilité), diminuant par là même le coût des puces, voire créant des fonctionnalités nouvelles tirant bénéfice de ses caractéristiques uniques.

\section{Les trois générations de MRAM}

Dans sa version actuelle, la cellule élémentaire d'une MRAM est une jonction TMR, couplée à un transistor de sélection permettant l'adressage aléatoire d'une cellule unique. Les cellules sont assemblées en blocs comprenant un grand nombre de cellules (par exemple $256 \mathrm{~K}$ ), entourés d'une électronique périphérique. La mémoire est complétée par les blocs d'alimentation et les plots de connexion avec le monde extérieur.

1 - Dans la première génération de MRAM, l'écriture utilise le champ magnétique généré par des matrices de lignes de courant placées à proximité des cellules magnétiques TMR. Afin de sélectionner une seule cellule, on utilise deux conducteurs croisés, l'un au-dessus, l'autre au-dessous de la cellule (fig. 5a). En tirant parti de la dépendance angulaire du champ de renversement de l'aimantation dans une nanostructure magnétique, seule la cellule se trouvant à l'intersection des deux lignes peut commuter.

Cette technique souffre malheureusement de problèmes intrinsèques:

- courant d'écriture élevé, empirant avec la miniaturisation ;

- erreurs d'écriture, c'est-à-dire commutation de cellules non sélectionnées et/ ou absence de commutation de cellules sélectionnées ;

- perte aléatoire et/ou irréversible de données, associée à une stabilité thermique (rétention dans le temps) insuffisante de l'aimantation.

Seul Everspin (ex-Freescale) continue dans cette voie, en proposant une technologie améliorée.

$\gg>$

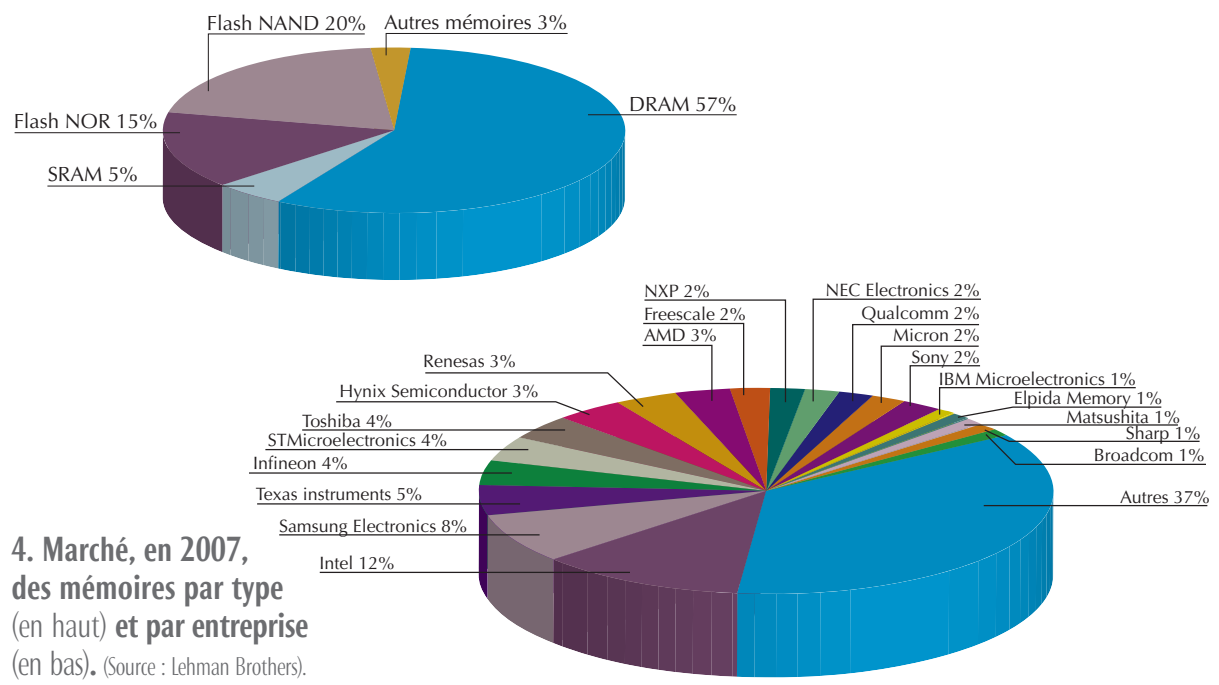

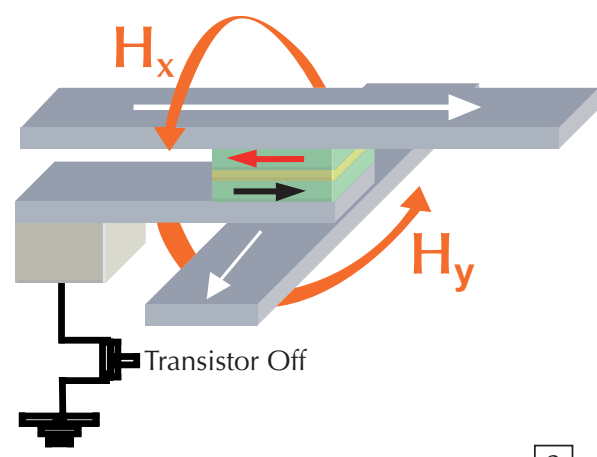

MRAM de première génération
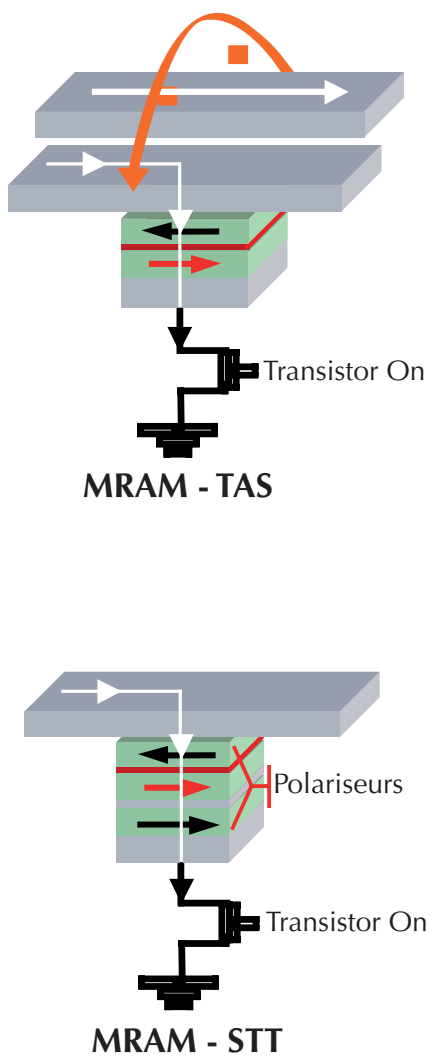

C

5. Principe de fonctionnement des trois générations de cellules MRAM.

(a) Les MRAM de première génération. L'écriture est assurée par l'envoi simultané de deux impulsions de courant (flèches blanches) dans deux lignes conductrices perpendiculaires, qui se croisent au niveau du point à adresser ; les champs magnétiques créés $\left(H_{x}\right.$ et $H_{y}$, flèches oranges) vont renverser l'aimantation d'une couche magnétique.

(b) Les MRAM-TAS. Un courant de chauffage (ligne blanche fine) permet d'obtenir le renversement de l'aimantation par un champ magnétique généré par un conducteur unique.

(c) Les MRAM-STI. Un courant polarisé en spin (ligne blanche fine) fait commuter l'aimantation d'une des couches magnétiques par un effet de transfert de spin, en l'absence de tout champ magnétique appliqué. 


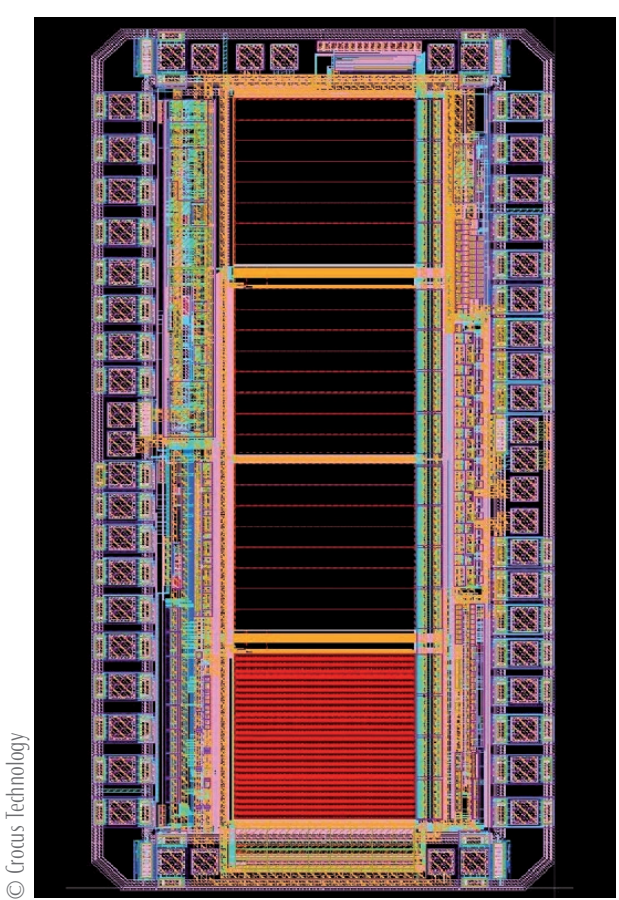

6. Technologie MRAM-TAS : mémoire $1 \mathrm{Mb}$.

$\gg>$

2 - Dans une variante améliorée, l'écriture est assistée par la température (TAS Thermally Assisted Switching) : simultanément à l'impulsion de champ magnétique, un courant est envoyé au travers du point mémoire via le transistor de sélection utilisé à la lecture (fig. 5b). Le chauffage induit au passage de la barrière tunnel (l'élément de plus forte résistance) permet d'abaisser le champ magnétique de renversement, facilitant ainsi l'écriture. Cette technique permet ainsi d'améliorer la sélectivité à l'écriture, tout en consommant une puissance réduite.

Dans la technologie développée par Crocus Technology (fig. 6), la couche de stockage est une bicouche ferromagnétique/ antiferromagnétique couplée par échange, avec une température critique $T_{b}$ à laquelle le couplage d'échange disparait. Dans ce cas, la cellule est chauffée audessus de $T_{b}$, température à laquelle il est possible de renverser l'aimantation, puis refroidie sous un champ magnétique d'écriture qui stabilise (" gèle ") l'aimantation dans la direction choisie.

Contrairement à la technologie Freescale, on découple dans cette approche la stabilité thermique (rétention) des données, pilotée par le couplage d'échange en dessous de $T_{b}$, de la puissance consommée à l'écriture au-dessus de $\mathrm{T}_{\mathrm{b}}$. Il est ainsi possible d'optimiser indépendamment les deux paramètres tout en obtenant une sélectivité parfaite, puisque seuls les bits sélectionnés sont chauffés et peuvent se renverser, quelle que soit l'amplitude du champ magnétique appliqué.

3 - L'effort de recherche dans le domaine des MRAM porte actuellement sur la technologie de transfert de spin (STTSpin Transfer Torque - voir Reflets n¹6, p. 9). Dans cette approche, l'écriture se fait uniquement par un courant polarisé en spin traversant le point mémoire via le transistor de sélection, sans champ magnétique appliqué (fig. 5c).

L'intérêt est évident : le paramètre pertinent pour le renversement de l'aimantation étant une densité de courant, le courant d'écriture décroît avec la taille de la cellule mémoire, facilitant la miniaturisation.

De plus, l'absence de ligne de génération de champ permet de superposer le point mémoire sur le transistor de sélection : la densité surfacique est alors potentiellement limitée seulement par la surface du transistor de sélection. Cette technologie est donc particulièrement attractive pour les applications envisagées aux nœuds technologiques avancés ( $\mathrm{F}=45 \mathrm{~nm}, 32 \mathrm{~nm} .$. ).

Malheureusement, pour être industrialisée cette technologie se heurte encore à deux problèmes fondamentaux :

- tout d'abord, le courant d'écriture est encore trop élevé, et sa valeur augmente fortement pour des impulsions de courant courtes (sub-10 ns). Il en résulte une dimension du transistor de sélection importante (pour pouvoir générer le courant requis) et donc, in fine, une densité de la mémoire limitée ;

- la seconde difficulté est d'assurer la stabilité thermique, donc la rétention de l'information, aux nœuds technologiques avancés. Une approche consiste à utiliser des jonctions tunnel magnétiques, dont l'aimantation est perpendiculaire au plan des couches. Cette configuration est plus favorable, puisqu'il est possible à courant d'écriture constant d'utiliser un matériau plus « dur » magnétiquement, donc plus stable. Malheureusement, les jonctions tunnel perpendiculaires ne sont pas encore au point, et il est difficile de combiner courant d'écriture faible et TMR élevée. Des progrès significatifs ont néanmoins été réalisés ces dernières années, et cette approche reste prometteuse.

La solution pourrait venir d'une combinaison de l'approche TAS (assistance thermique) avec l'écriture par transfert de $\operatorname{spin}(\mathrm{STT})$. Que ce soit en géométrie planaire ou perpendiculaire, cette approche est la seule permettant d'atteindre les nœuds technologiques sub-32 nm puisque, comme en TAS " classique » (avec champ magnétique), la stabilité thermique est obtenue par le couplage d'échange et découplée du courant d'écriture. Le calcul donne avec les matériaux actuels utilisés dans la mémoire TAS "classique " une limite de stabilité théorique autour de $20 \mathrm{~nm}$. Les premiers résultats obtenus par Crocus Technology sont très prometteurs.

\section{Conclusion}

Le marché des têtes de lecture pour disque dur est toujours en croissance, avec l'explosion du numérique et les besoins de stockage associés. Cependant, une tête de lecture, fondamentalement un capteur de champ (voir article de C. Fermon, p. 8), reste in fine un composant élémentaire. C'est d'ailleurs ce qui a permis l'essor industriel rapide, puisqu'il est possible de " trier » sur un même substrat les bons et les mauvais éléments, permettant ainsi d'obtenir immédiatement des produits (et donc des revenus !) malgré des rendements de fabrication médiocres.

Il est encore trop tôt pour savoir si la MRAM sera un succès commercial ou simplement une technologie prometteuse confinée à des marchés de niche. Au regard des annonces de produits et d'applications qui paraissent de plus en plus régulièrement dans la presse, de la maturité de la technologie et de l'intérêt suscité par les concepteurs de circuits et systèmes, il semble cependant que la MRAM soit en train de trouver son public. En composant discret dans des applications de type "SRAM non volatile ", en mémoire embarquée dans des circuits logiques en remplacement des Flash ou des SRAM, voire purement et simplement en substitution possible aux DRAM, la MRAM devrait dans les années à venir gagner des parts de marché significatives.

Pour l'électronique de spin, l'étape suivante consistera alors à intégrer des points mémoires magnétiques au cœur même des circuits logiques, pour aboutir au concept de circuit logique magnétique reprogrammable. Les chercheurs travaillent sur ces objectifs et des premiers circuits simples intégrant des cellules magnétiques ont déjà vu le jour. I 